\title{
Noise cancellation using total variation for copy number variation detection
}

\author{
Fatima Zare $^{1 *}$, Abdelrahman Hosny ${ }^{1}$ and Sheida Nabavi ${ }^{2}$ \\ From the 6th Workshop on Computational Advances in Molecular Epidemiology (CAME 2017) \\ Boston, MA, USA. 20 August 2017
}

\begin{abstract}
Background: Due to recent advances in sequencing technologies, sequence-based analysis has been widely applied to detecting copy number variations (CNVs). There are several techniques for identifying CNVs using next generation sequencing (NGS) data, however methods employing depth of coverage or read depth (RD) have recently become a main technique to identify CNVs. The main assumption of the RD-based CNV detection methods is that the readcount value at a specific genomic location is correlated with the copy number at that location. However, readcount data's noise and biases distort the association between the readcounts and copy numbers. For more accurate CNV identification, these biases and noise need to be mitigated. In this work, to detect CNVs more precisely and efficiently we propose a novel denoising method based on the total variation approach and the Taut String algorithm.
\end{abstract}

Results: To investigate the performance of the proposed denoising method, we computed sensitivities, false discovery rates and specificities of CNV detection when employing denoising, using both simulated and real data. We also compared the performance of the proposed denoising method, Taut String, with that of the commonly used approaches such as moving average (MA) and discrete wavelet transforms (DWT) in terms of sensitivity of detecting true CNVs and time complexity. The results show that Taut String works better than DWT and MA and has a better power to identify very narrow CNVs. The ability of Taut String denoising in preserving CNV segments' breakpoints and narrow CNVs increases the detection accuracy of segmentation algorithms, resulting in higher sensitivities and lower false discovery rates.

Conclusions: In this study, we proposed a new denoising method for sequence-based CNV detection based on a signal processing technique. Existing CNV detection algorithms identify many false CNV segments and fail in detecting short CNV segments due to noise and biases. Employing an effective and efficient denoising method can significantly enhance the detection accuracy of the CNV segmentation algorithms. Advanced denoising methods from the signal processing field can be employed to implement such algorithms. We showed that non-linear denoising methods that consider sparsity and piecewise constant characteristics of CNV data result in better performance in CNV detection.

Keywords: Next generation sequencing, Copy number variation, Signal processing, Total variation, Taut string, Denoising

\footnotetext{
* Correspondence: fatemeh.zare@uconn.edu

${ }^{1}$ Computer Science and Engineering Department, University of Connecticut,

Storrs, CT, USA

Full list of author information is available at the end of the article
}

(c) The Author(s). 2018 Open Access This article is distributed under the terms of the Creative Commons Attribution 4.0 International License (http://creativecommons.org/licenses/by/4.0/), which permits unrestricted use, distribution, and reproduction in any medium, provided you give appropriate credit to the original author(s) and the source, provide a link to the Creative Commons license, and indicate if changes were made. The Creative Commons Public Domain Dedication waiver (http://creativecommons.org/publicdomain/zero/1.0/) applies to the data made available in this article, unless otherwise stated. 


\section{Background}

Understating the inherited basis of genomic variations and their contribution to phenotypes is the major goal of genomics. One of the main types of genomic variation is copy number variation (CNV), defined as a phenomenon in which sections of a genome, ranging from a few hundred base pairs to a few mega base pairs, are repeated or deleted $[1,2]$. It is observed that $4.8-9.5 \%$ of the genome contributes to $\mathrm{CNV}$ and they affect more nucleotides per genome compared to single nucleotide polymorphism (SNP) [3]. CNVs can change gene dosage, create new genes, reshape gene structures, and modify gene expression regulatory elements $[4,5]$, and as a result they can significantly influence gene expression and phenotypic variation [6]. CNVs, particularly exon rearranging and gene duplication, can be a major procedure driving gene and genome evolution [7]. CNVs are associated with genetic disease susceptibility [7, 8], evolution and normal phenotypic variation. Recently several studies have indicated that there is a relationship between CNVs and many diseases including cancer [9]. CNV assessment is also important in functional genomic studies since not considering CNVs can result in misinterpretation of gene expression, methylation or chromatin immunoprecipitation data $[10,11]$. The power to discover a relationship between genomic variation and phenotype is limited by the sensitivity, accuracy and comprehensibility of genomic variation identification methods. As a result, precise and efficient detection of CNVs, and accessible CNV detection software tools are very important in the advancement of genomics.

For studying CNVs, using array-based technologies has been a popular approach since late 1990s due to their reasonable cost and relatively high resolution. [12]. With the arrival of next generation sequencing (NGS) technologies [13] in the late 2000s and early 2010s; and because of limitations of array-based technology associated with hybridization and resolution, sequence-based $\mathrm{CNV}$ detection has become a more popular approach to detect CNVs with higher accuracy and resolution [14]. Consequently, several computational tools have been developed to identify CNVs using NGS data. However, accurate detection of CNVs from NGS data remains challenging [15] for a variety of reasons, including the big data nature of the NGS data, short-read lengths, sequence-specific biases, library preparation biases, and high level of noise. Comparative analyses of the performance of the CNV identification tools show that the tools' false positive rates are high and agreements across the tools is low [16-18].

In general, there are four major methods to detect CNVs from NGS data: 1) read depth, 2) paired-end reads, 3) split reads, and 4) assembly [19-21]. Compared to other methods, RD-based methods can identify the exact number of CNVs, as the paired-end and split read approaches can only detect the position of the potential CNVs and not the copy numbers. Furthermore, RDbased approaches can work better on large sized CNVs, which are hard to detect by the paired-end and split read approaches [22]. Assembly-based methods are used less often in CNV detection because they are computationally very demanding. Furthermore, eukaryotic genomes contain a remarkable segmental duplication that render poor performance of assembly-based methods in these complex regions. Another problem with assembly-based approaches is that they fail to handle haplotype sequences and as a result only homozygous structural variations can be identified [23]. With availability of high-coverage NGS data and because of the above reasons, RD-based approaches have recently become a main method to detect CNVs, particularly for targeted sequencing data such as whole exome sequencing (WES) data. Mostly, in the RD-based approach, a non-overlapping sliding window is utilized to measure the number of reads that are aligned to a genomic region overlapped with the window. It is hypothesized that the number of short sequences that align to a position in the genome (readcount) is proportional to the copy number at that position. The readcount values, are used to detect CNV regions using segmentation methods [24-34]. However, existence of biases and noise distorts the correlation between copy numbers and the readcount values, which reduces the RD-based methods' ability to detect CNVs accurately. Hence, a robust CNV detection method requires elimination of biases and noise from data before detecting CNVs. In general RD-based approaches include two main steps: 1) preprocessing, and 2) segmentation. In the preprocessing step, readcount data are generated, low quality read counts are removed, and readcount data are normalized to reduce bias. Even though readcount data is very noisy, most of the $\mathrm{CNV}$ detection methods do not employ denoising as part of their preprocessing step.

There are several sources for biases and noise in NGS data such as GC bias, mappability bias, sample contamination, sequencing noise, and experimental noise. GC content has been found to affect read coverage on most sequencing platforms and varies significantly along the genome [35-37]. Due to biochemical differences in the sequenced DNA, sequencing technologies act differently on sequences with different GC content [38]. It has been shown that segments of genome with low or high GC content have low readcounts compared to other segments. As a result, there is a unimodal correlation between readcounts and $\mathrm{G}$ and $\mathrm{C}$ bases in the genome [39-42]. Although the global structure of the distribution of readcounts with respect to the GC content (GC bias curve) is consistent, the exact structure differs remarkably across samples. Several methods have been 
proposed to remove $\mathrm{GC}$ bias, the most popular of which is the Loess regression method [41, 43, 44].

In addition, because of short length of reads and the existence of repetitive regions within the reference genome, a huge number of NGS reads cannot be clearly mapped to the reference genome. Especially for WES data, some regions of the genome have low or no coverage. Sequencing errors and mutations can lead to incorrectly mapped reads as well. These errors introduce a challenge to the alignment process resulting in a mappability bias [15]. To reduce mappability biases, CNV detection approaches typically utilize the number of uniquely mapped short reads in a sample and a normal reference and apply a Loess regression method $[45,46]$. To compensate for GC and mappability biases, many RD-based methods $[26,33,34,44]$ use the ratio of sample readcounts to normal reference readcounts.

Most of the CNV detection tools focus only on reducing GC and mappability biases, which do not represent all types of possible noise, and thus considerable amount of noise remains untouched after normalization. A few $\mathrm{CNV}$ detection tools employ denoising techniques such as Bayesian approaches [28] and the discrete wavelet transform (DWT) [47] to reduce noise from readcount data. Signal processing techniques have been widely used for effective noise reduction. These techniques are broadly utilized to improve signal-to-noise ratio (SNR) in engineering where signals are a mixture of the original signals and various types of complex noise. However, they have had a very limited application in genomics [48-50]. Readcount data can be seen as a noisy signal with some characteristics. First, it is sparse, that means the total length of CNVs is much less that the total length of genome. Second, since copy numbers are discrete values, it is piecewise constant signal. Due to the importance of the breakpoints of CNVs, denoising methods that can preserve edges need to be used. Also, a very challenging issue in $\mathrm{CNV}$ identification is the difficulty of detecting focal (narrow aberration) CNV regions in the presence of extreme noise.

Because of the characteristics of readcount data and the need for the accurate detection of breakpoints and focal CNVs, in this work we used a total variation approach for denoising. In signal processing, total variation $[51,52]$ approaches have been very successful in removing noise from a noisy sparse piecewise constant signal while preserving edges. A noisy signal contains many unwanted details that lead to high total variation that is the summation of the absolute gradient of the noisy signal, while has few breakpoints. Therefore, a close match to the original signal can be estimated by minimizing the total variation of the signal. This optimization approach can remove unnecessary details of the noisy signal and at the same time preserve important ones such as breakpoints and narrow changes. A very efficient implementation of total variation denoising is Taut String [53], which solves the optimization problem in a non-iterative in-place manner.

The main goal of this study is to develop an efficient and effective denoising algorithm to remove biases and noise from readcount data for better identification of CNVs using NGS data. In this work, we introduced an efficient and accurate denoising technique based on a signal processing approach, Taut String [53-55]. This approach efficiently removes noise while preserves breakpoints and prepares error free readcount data for the segmentation.

\section{Methods}

In this study, we use sparse and piecewise constant characteristics of $\mathrm{CNV}$ signal to reduce readcount data noise. We developed a denoising algorithm based on the Taut String approach. Before applying denoising we first filtered low quality readcount data and removed GC and mappability bias from readcount data [56]. After applying denoising, a segmentation method was used to call $\mathrm{CNV}$ regions from denoised readcount data. We applied the circular binary segmentation (CBS) algorithm [57] for segmentation.

\section{Filtering low quality readcounts}

We applied a sliding window approach to compute the $\mathrm{GC} \%$ and readcount value for each genomic window with an optional size [58]. In this work, the size of windows is $100 \mathrm{bp}$. We considered windows with readcounts and GC content in the bottom and top $1 \%$ percentiles as outliers and removed them.

\section{Reducing bias}

Several methods have been proposed for modeling and removing GC and mappability biases from data [43, 45, $59,60]$. In order to remove GC bias, we followed the weighted Loess regression method proposed in [43]. In this method, a local weighted regression is applied to the means of the number of reads mapped to windows with a GC content of $g c$ (percentage of $\mathrm{G}$ and $\mathrm{C}$ bases for each window), $m_{g c} \mathrm{~s}$ [43]. It is observed that if there are a few windows with a GC content of $g c$, then their corresponding $m_{g c}$ values would be significantly higher or lower than $m_{g c}$ values corresponding to other windows. The weighted Loess regression method tries to remove these local extremes, resulting in smoother values of $m_{g c}$. Then, using the smoothed $m_{g c} s$, the number of reads for each window will be corrected.

After applying GC bias correction on both sample and normal readcount data, we compute the ratio of sample to normal readcount for each window. These ratios can also help to mitigate mappability bias. 


\section{Reducing noise using taut string}

The accuracy of CNVs detection is heavily influenced by the noisiness of the readcount data that can be considered as readcount signals. It is observed that under highly noisy readcount signals, CNV detection tools identify many false CNVs (false positives (FPs) and false negatives (FNs)). Therefore, reducing noise is an essential step in a CNV detection algorithm.

The $\log 2$ ratios of sample and normal readcounts can be modeled as Eq. (1):

$$
\mathbf{r}=\mathbf{f}+\boldsymbol{\varepsilon},
$$

where $\boldsymbol{\varepsilon}$ indicates noise and is defined as a vector of independent and identically distributed (iid) random variables with a normal distribution $N\left(0, \sigma_{N}^{2}\right)$ (mean of 0 and standard deviation of $\sigma_{N}$ ). A denoising method tries to recover the original signal $\mathbf{f}$ from the noisy observed signal $\mathbf{r}$.

There are several approaches for removing noise from noisy signal. The characteristics of the noise and signal should be considered for developing an appropriate noise cancelation method. Fourier based filtering techniques [61] and Kernel estimators [62] are identified as two popular approaches for removing noise. However, when the noise and signal Fourier spectra overlap, these methods cannot separate spectra completely and fail to detect original signal [63]. Identifying small CNV segments is another challenge in a noisy environment. Usually, linear denoising approaches cannot perform well in detecting small CNV segments in low SNR environments. For a noisy readcount signal, amplitude distortion happens more often than spectra location distortion. In this situation, non-linear approaches that consider amplitudes rather than locations of the spectra in their noise cancelation procedure perform better. Furthermore, accurate detection of breakpoints plays an important role in preserving narrow $\mathrm{CNVs}$ while removing noise.

In this study, for estimating $\mathbf{f}$ from given noisy readcount data/signal, we employed an effective and efficient non-linear noise cancelation approach based on the total variation denoising for one-dimensional (1-D) discrete signals $[53,54]$ that can preserve edges and narrow segments. The total variation denoising has the ability to identify local extreme values in data with low SNR by estimating a piecewise constant signal $[64,65]$.

Given a noisy signal $\mathbf{r}=\left(r_{1}, r_{2}, \ldots, r_{n}\right)$, the goal is to estimate the denoised signal $\hat{\mathbf{f}}$ which minimizes the eq. (2).

$$
\min _{f} \frac{1}{2} \sum_{i=1}^{n}\left|r_{i}-f_{i}\right|^{2}+\lambda \sum_{i=1}^{n-1}\left|f_{i+1}-f_{i}\right|
$$

for some regularization parameter $\lambda \geq 0$. The first term is used to measure the fitting error between noisy signal $r_{i}$ and denoised signal $f_{i}$, and the second term is used to measure the penalty caused by the difference between change-points $f_{i}$ and $f_{i+1}$ using a sparsity-inducing regularizer $(\lambda)$. The challenging part is selecting appropriate value for $\lambda$. In [54], $\lambda$ is chosen as $\lambda=c n^{\frac{1}{2}} \sigma$ for some $C>0$, where $\sigma$ is computed as $\sigma=1.48 / \sqrt{ } 2\left\{\left|r_{2}-r_{1}\right|\right.$, $\left.\ldots,\left|r_{n}-r_{n-1}\right|\right\}$.

This optimization problem can generate a piecewise constant signal whose number of breakpoints, $k$, is a non-increasing function of $\lambda$ [64]. It considers the smallest integer $k$ and tries to find $\hat{\mathbf{f}}$ with $k$ local extreme values.

To solve this total variation optimization problem, we used the efficient Taut String approach. Taut String is an in-place non-iterative linear time method for 1-D TV denoising. Taut String defines a vector of running sums $R_{i}=\sum_{u=1}^{i} r_{u}, 1 \leq i \leq \mathrm{n}$. Then equation (2) is converted to equation (3) using $f_{i}=s_{i}-s_{i-1}$ for $1 \leq i \leq n$ [66].

$$
\begin{gathered}
\min _{s \in \mathbb{R}^{n+1}} \sum_{i=1}^{n} \sqrt{1+\left|s_{i}-s_{i-1}\right|^{2}} \text { subject to } \\
s_{0}=0, s_{n}=R_{n} \text {, and } \max _{1 \leq i \leq n}\left|s_{i}-R_{i}\right| \leq \lambda
\end{gathered}
$$

To minimize equation (3), suppose a tube of radius $\vartheta=0.5 \lambda$ consists of the lower bound $l_{i}:=R_{i}-\vartheta$ and the upper bound $u_{i}:=R_{i}+\vartheta$. Then, assume that there exist a string connecting $\left(1, R_{1}\right)$ and $\left(\mathrm{n}, R_{n}\right)$, restrained to lie within the tube, and it is pulled to the point that it is tight, touching the tube (at possibly multiple "knots") on either side. Taut String tries to solve this problem by using the greatest convex minorant and least concave majorant of the upper and lower strings $R_{i}+\vartheta$ and $R_{i}-\vartheta$. The solution of this optimization, $\hat{\mathbf{f}}$, can be considered as a string between $R_{i}-\vartheta$ and $R_{i}+\vartheta$ that is pulled tight.

To improve the convergence rate at local extremes, we used the method introduced by Davies and Kovac [54] that combines the Taut String with a multiresolution bound over estimated residuals and utilizes an additional local squeezing step to the Taut String estimate. In this approach, Taut String checks if the deviation between the observed data and $\hat{\mathbf{f}}$ satisfy the multiresolution criterion [54], if not, it uses local squeezing of tube. It means that it squeezes the tube by multiply a value $\gamma \in(0,1)$ to $\lambda$ and obtains new upper and lower bound. This approach starts from the fixed point $s_{0}=0$. It gradually calculates the greatest convex minorant of the upper bounds, and the smallest concave majorant of the lower bounds on the tube. A segment of the Taut String can be detected when both curves intersect. Then, the algorithm is restarted at the end of the detecting segment and is run until all Taut String segments have been identified. By employing this approach, computing $\hat{\mathbf{f}}$ is an efficient procedure and it is linear in time $(O(n))$ [54]. This algorithm yields piecewise constant functions. On 

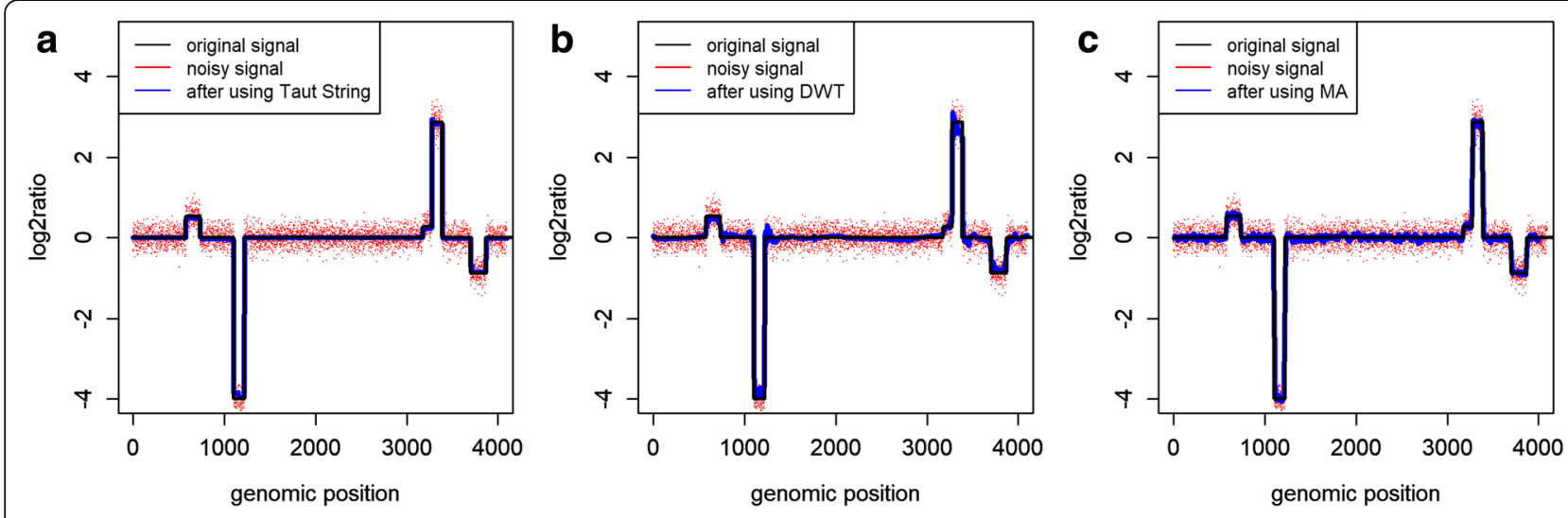

Fig. 1 Denoising with a) Taut String and b) DWT c) MA. Using simulated readcount data at SNR =7

each constant interval, the denoised values is equivalent to the mean of the corresponding observations, except for local extrema of the fit. This algorithm removes very low-frequency noise while keeping the location of change-points (breakpoints).

\section{Data sets}

\section{Simulated readcount data sets}

We generated simulated data to evaluate the performance of our proposed denoising method in identifying true CNVs and their breakpoints. For this purpose, 50 simulated readcount signals were generated with known true CNV segments as gold standard. Each data point represents a readcount value of a genomic window. By adding different levels of Gaussian white noise to these readcount data, we generated noisy readcount signals with different SNRs, where SNR is defined as $S N R=\left(P_{-}\right.$original signal $) /\left(P_{-}\right.$noise $)$. A Gaussian white noise has a perfectly flat power with $P_{\text {noise }}=\sigma_{N}^{2}$.

\section{Simulated sequencing data sets}

We used simulated WES data sets with known true CNVs as gold standard. The simulated data were generated by CNV-Sim tool (https://github.com/NabaviLab/ CNV-Sim). We generated 10 data sets using CNV-Sim for chromosome 1 . We aligned simulated short reads to the reference genome (hg19) using the BWA software tool [67]. We generated readcounts from aligned sequencing data by utilizing the bedtools suit [58] and employing100bp sliding window. We used these simulated data to investigate the performance of the CNV detection tools in terms of sensitivity, false discovery rate, and specificity.

\section{Real data sets}

For this work, we downloaded 10 Breast cancer tumor and matched normal WES data from the Cancer Genome Hub (https://cghub.ucsc.edu/index.html). We used BWA to align these sequencing data. The bedtools suit [58] and $100 \mathrm{bp}$ sliding window were utilized to generate readcount signals from the aligned sequencing data. The
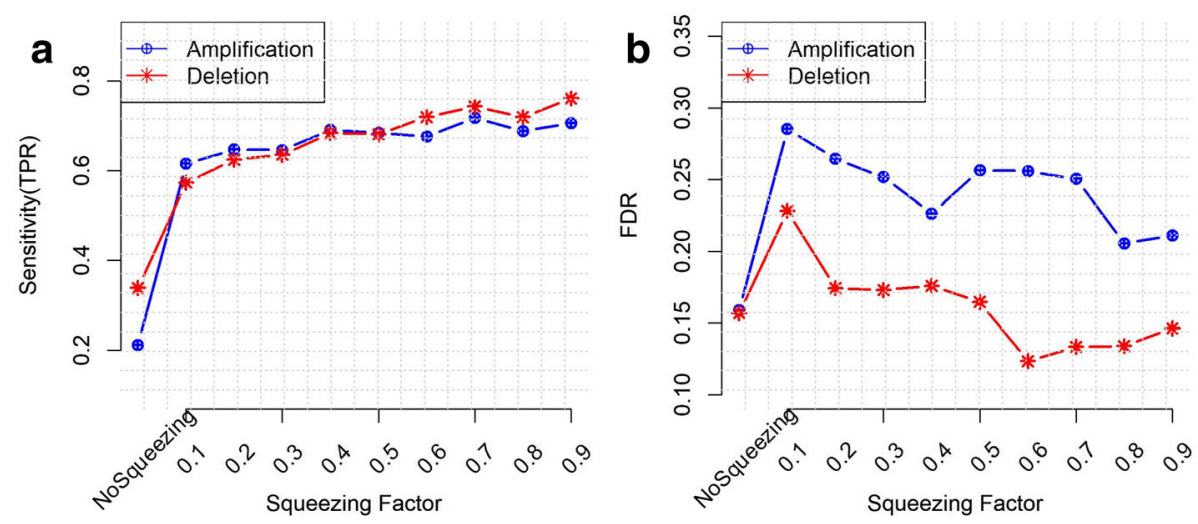

Fig. 2 The effect of the squeezing factor on a) Sensitivity b) FDR of CNVs detection. Using simulated readcount data 

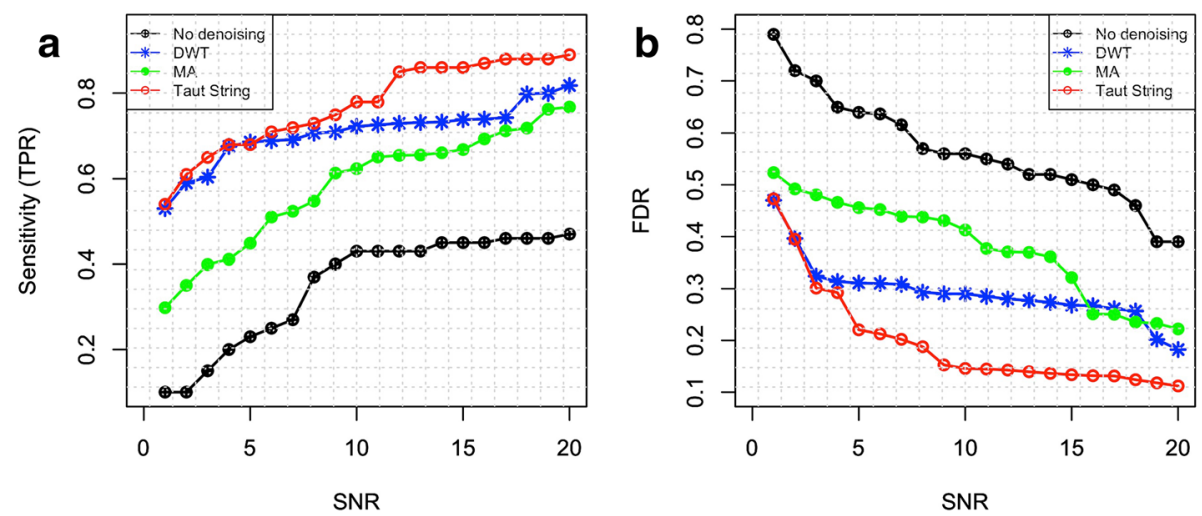

Fig. 3 a) Sensitivity and b) FDR of detection of amplified CNVs segments before and after applying denoising methods for different SNR. Using Simulated Readcount data

array-based CNV results from the same samples provided by the cancer genome atlas (TCGA) were used for benchmarking and evaluating the performance of the denoising methods in terms of sensitivity, false discovery rate, and specificity. We downloaded the $\mathrm{CNV}$ results from the SNP array platform from the Genomic Data Commons data portal (https://portal.gdc.cancer.gov).

\section{Results}

\section{Simulated readcount data}

In this section, we compared the performance of Taut String, with DWT and moving average (MA) denoising approaches. Figure 1 shows the simulated readcount signal before and after applying denoising methods on a noisy signal with SNR $=7$. It can be seen that Taut String works better compared to DWT and MA in preserving edges and generating piecewise constant data. To compare the performance of these denoising methods in more detail, we computed sensitivities and false discovery rates (FDRs) of calling CNV segments applying the CBS segmentation. Also, we computed the breakpoint accuracy of the detected $\mathrm{CNV}$ segments using the denoising methods at different levels of noise. We investigated the effect of Taut String in calling true CNVs with different lengths. Furthermore, we investigated the detection power of true CNV using the Taut String denoising with different values of local squeezing $\gamma$.

\section{The effect of the squeezing factor $\gamma$ on the taut string performance}

We evaluated the performance of Taut String in terms of sensitivity in detecting true CNVs using different values of the squeezing factor $\gamma$.We used the 50 simulated readcount data sets that we explained above. Figure 2 shows that the sensitivities in calling $\mathrm{CNV}$ segments improve by using squeezing factors close to 1 . We can see that selecting an appropriate squeezing factor is important. When $\gamma$ is small the algorithm ends rapidly as all multiresolution coefficients satisfy the multiresolution criterion quickly and generate many local extreme values. Some of them are outlier and lead to detect more FP CNVs. When $\gamma$ is nearly 1 , the algorithm takes more time and identifies smaller number of local extreme values. Applying Taut String without using the squeezing factor results in lower
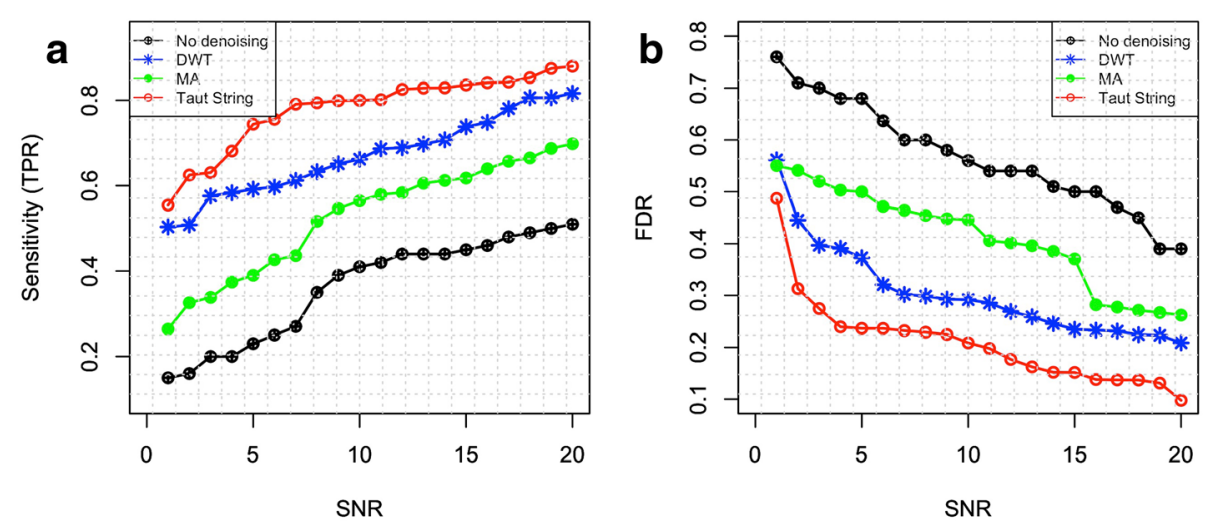

Fig. 4 a) Sensitivity and b) FDR of detection of deleted CNVs segments before and after applying denoising methods for different SNR. Using simulated Readcount data 


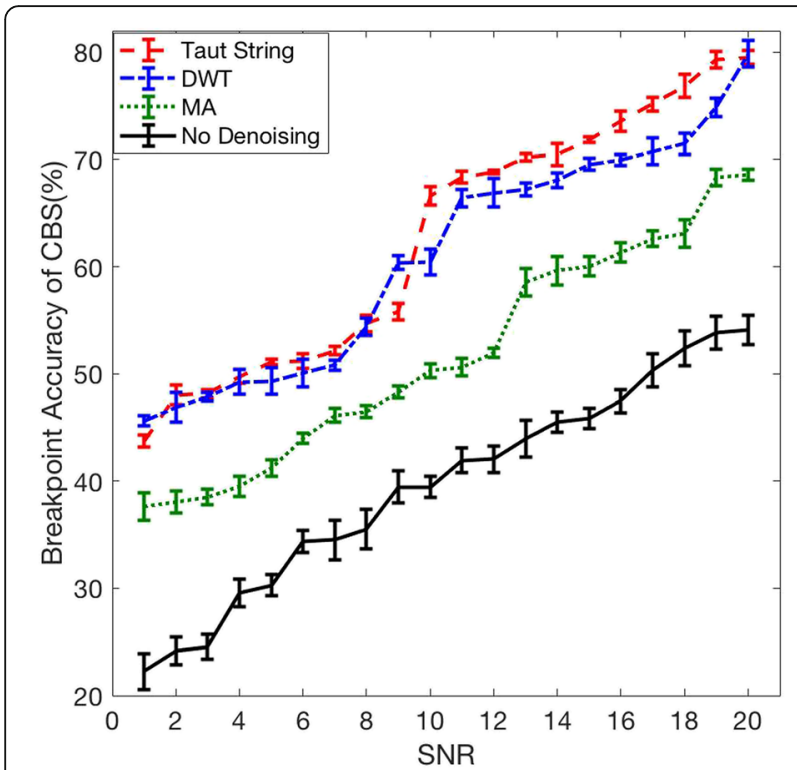

Fig. 5 Breakpoint accuracy before and after applying denoising for different SNR, using simulated readcount data

sensitivity and lower FDR compared to using the squeezing factor. Considering a tradeoff between time cost and accurate detection of CNVs segments, we chose $\gamma=0.5$ for the rest of our work.

\section{Sensitivity and FDR of identifying CNV segments}

Using the 50 sets of simulated readcount signals with different levels of noise ( $\sigma_{N}$ from 0.47 to 0.05 ), we compared the sensitivities in detecting CNV segments using DWT, Taut String and MA. Segment-based comparison [18], which considers the overlap between detected CNVs regions and benchmark CNVs, was used to compute true positives (TPs), FN, and FPs. The segment-based FPs and TPs were used to calculate sensitivities, specificities and FDRs. "GenomicRanges" $\mathrm{R}$ package from Bioconductor [68] is applied to calculate overlapping regions between detected CNVs and benchmark CNVs. A threshold of \pm thr for $\log _{2}$ ratios was used for calling CNV segments. TP happens when a detected amplified/deleted segment has an overlap of $80 \%$ or more with a benchmark amplified/ deleted segment. FN happens when an amplified/deleted segment in the benchmark does not have an overlap of $80 \%$ or more with any detected amplified/deleted regions. FP happens if there is no overlap of $80 \%$ or more between a detected CNV region and any benchmark CNVs. Sensitivity and FDR are defined as $T P /(F N+T P)$ and $F P /(F P+$ $T P$ ), respectively.

Figures 3 and 4 show the sensitivity and FDR of detecting amplified and deleted segments, respectively, with $t h r=0.2$. It can be seen that all three denoising methods improve sensitivity and FDR of CNV detection. However, edge protecting approaches (Taut String and DWT) can improve sensitivity and FDR significantly better than MA, and Taut String outperforms DWT in detecting CNVs. All three methods perform better for higher SNRs. Even at a high SNR condition, the segmentation algorithm without denoising provides so many false detections, where employing Taut String can result in near perfect detection.

\section{Breakpoint accuracy in different level of noise}

The three denoising methods DWT, MA and Taut String were applied to the 50 simulated readcount data with different level of SNRs ranging from 1 to $20\left(\sigma_{N}\right.$ from 0.47 to 0.05 ). Then, CNVs' segments were identified from the denoised and noisy readcount data by using CBS. We defined breakpoint accuracy as the frequency of identifying exactly true start and end points of detected CNVs' segments. We used the known start and end points of simulated CNVs' segments to compute these frequencies. Figure 5 shows the performance of DWT, Taut String and MA in detecting breakpoints. As also depicted in Fig. 1, we can observe that using an appropriate smoothing approach before segmentation improves the breakpoint accuracy significantly. It can be seen that DWT and Taut String outperform MA. At
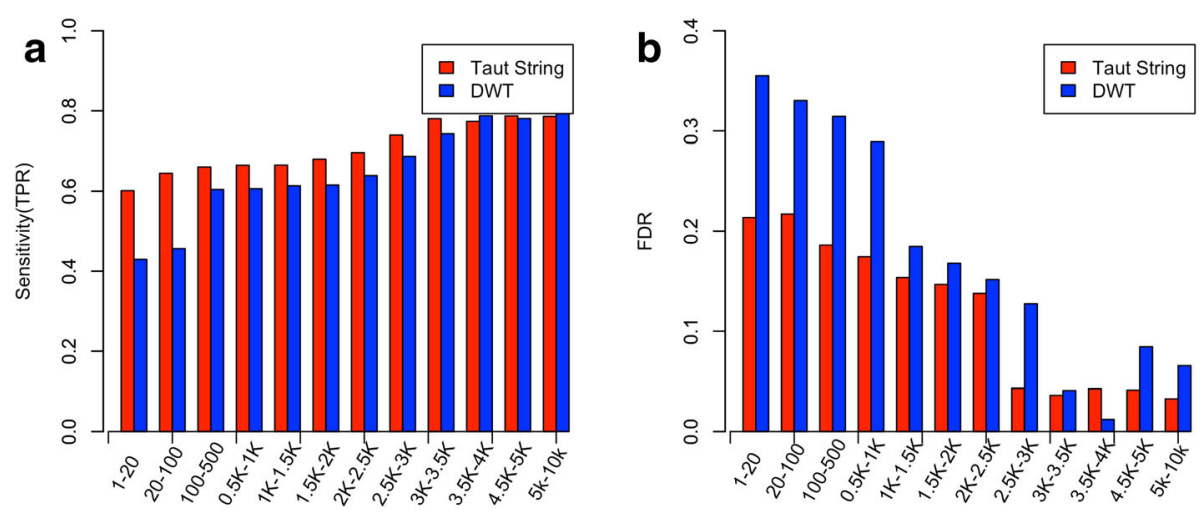

Fig. 6 Sensitivity and FDR of detection of amplified CNV segments with different CNV lengths 
lower levels of SNR (higher noise), DWT and Taut String perform almost similar but at higher levels of SNRs Taut String performs slightly better than DWT. For having a high accuracy of CNV breakpoints detection, the denoising method should provide sharp edges with less fluctuation around the edges. Denoised signals using Taut String show less fluctuation at the breakpoints compared to DWT and MA (Fig. 1). Taut String denoising is more powerful to preserve edges leading to better performance in breakpoint detection.

\section{The effect of denoising methods in detecting CNVs with different lengths}

Each data point in simulated data represents a readcount value of a sliding window. Simulated data sets contain $\mathrm{CNV}$ segments with different length (in window size) ranging from 1 to $10 \mathrm{k}$. Figures 6 and 7 show the sensitivities and FDRs in detecting CNVs with different length using Taut String and DWT. We can see that in general sensitivity and FDR is better in detecting larger CNVs. However Taut String outperforms DWT for narrower CNVs. For CNV segments with lengths between 1 and 20 , sensitivity in detecting amplification/deletion is $0.60 / 0.66$ when using Taut String, and is $0.42 / 0.34$ when using DWT. We can see a stronger pattern in FDRs. For CNV segments with lengths between 1 and 20, FDR in detecting amplification/deletion is $0.21 / 0.18$ when using Taut String and is 0.35/0.32 when using DWT.

\section{Simulated sequencing data using CNV-Sim}

As explained above, we generated 10 simulated pairedend WES data sets with read length of $100 \mathrm{bp}$ for chromosome 1 using the CNV-Sim simulator. Using simulated sequencing reads from genomes that contain known CNVs, we calculated sensitivities and FDRs of detecting CNVs with and without using denoising. We used the lists of known simulated CNV for benchmarking.
Table 1 Possible results for each candidate CNV genes

\begin{tabular}{lll}
\hline CNV gene & Not identified & Identified \\
\hline Present & FN & TP \\
Not present & TN & FP \\
Performance metrics: & & \\
Sensitivity $=\frac{T P}{F N+T P}$ & FDR $=\frac{F P}{F P+T P}$ & Specificity $=\frac{T N}{F P+T N}$ \\
\hline
\end{tabular}

To calculate Sensitivity and FDR, we used a genebased approach [18]. First, we used CBS to call CNV segments and then we annotated the identified CNV segments to derive CNV gene lists. We used "cghMCR" $R$ package from Bioconductor [69] to identify CNV genes using Refseq gene identifications. A threshold of \pm thr for $\log 2$ ratios was used to detect CNV genes, that is: amplification for $\log 2$ ratios $>t h r$, deletion for $\log 2 \mathrm{ra}$ tios $<-t h r$, and No CNV for $\log 2$ ratios between $-t h r$ and $t h r$. Here, we used $t h r=0.5$. Table 1 shows definitions for sensitivity (true positive rate (TPR)), FDR or 1-Precision, and specificity (SPC). Table 2 shows overall performance of the denoising methods using the simulated short read data.

Similar to simulated readcount data we observed that denoising improves the performance of $\mathrm{CNV}$ identification; and Taut String outperforms DWT. Using the Taut String denoising, the sensitivity of detecting amplifications improves from 79.65 to $87.17 \%$ and the sensitivity of detecting deletions improves from 78.64 to $88.15 \%$.

\section{Real data}

To investigate the performance of the denoising methods in identifying CNVs, we evaluated the results of CNV detection with and without applying denoising methods using the real data sets. The results are shown in Table 3. It can be seen that denoising methods improve the performance of the CNV detection. In overall, by using the Taut String denoising method, the sensitivity of detecting amplifications improves from 50.99 to $69.52 \%$ and the sensitivity of detecting deletions improves from 60.37 to
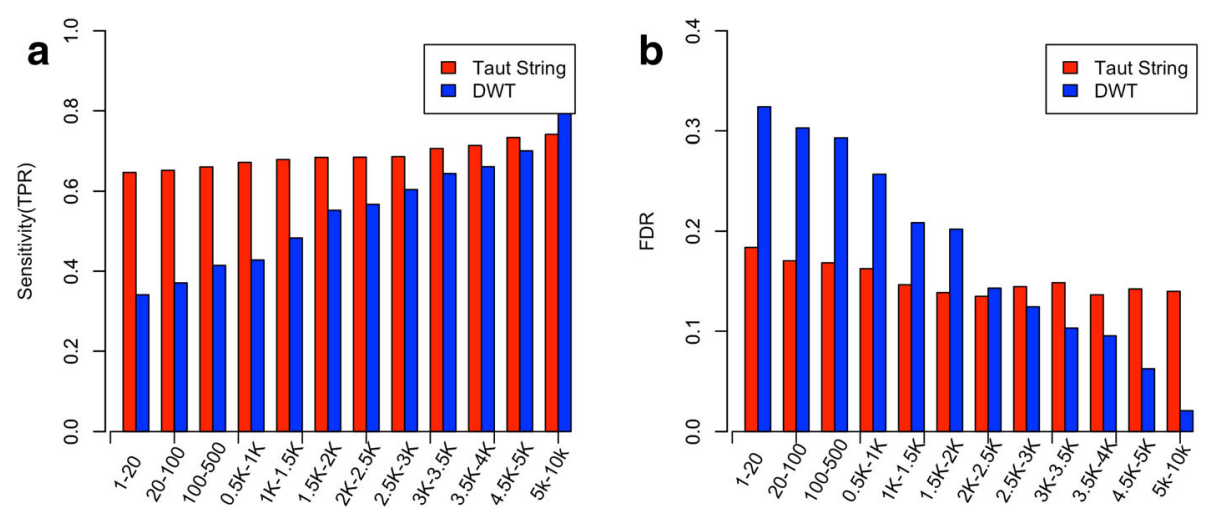

Fig. 7 Sensitivity and FDR of detection of deleted CNV segments with different CNV lengths 
Table 2 Overall Performance of The Denoising Methods Using the simulated WES data generated by CNV-Sim Data

\begin{tabular}{llllllll}
\hline Denoising Methods & Amplified CNVs & & & \multicolumn{3}{l}{ Deleted CNVs } \\
\cline { 2 - 3 } \cline { 6 - 8 } & Sensitivity & FDR & Specificity & & Sensitivity & FDR & Specificity \\
\hline Before applying denoising method & $79.65 \%$ & $35.23 \%$ & $80.93 \%$ & & $78.64 \%$ & $37.03 \%$ & $81.02 \%$ \\
After applying DWT & $86.87 \%$ & $22.88 \%$ & $91.32 \%$ & & $87.20 \%$ & $20.54 \%$ & $90.32 \%$ \\
After applying Taut String & $87.17 \%$ & $22.94 \%$ & $92.82 \%$ & & $88.15 \%$ & $23.65 \%$ & $89.49 \%$ \\
\hline
\end{tabular}

79.93\%. As expected, Taut String works better than DWT in denoising real readcount data results in higher sensitivity and specificity in CNV detection. Removing noise by Taut String and DWT, increases the number of both TP and TN and decreases the number of both FP and FN leading to improving the overall performance of a $\mathrm{CNV}$ detection method.

Using the real data sets, we compared the copy number values, in $\log 2$ ratios, of detected $\mathrm{CNV}$ genes with those of their corresponding benchmark CNV genes, before and after applying Taut String and DWT. Results show that after using Taut String, copy number values of $76.87 \%$ of amplified genes and $70.26 \%$ of deleted genes differ from their benchmark copy number values less than $20 \%$ of the benchmark copy number values. Table 4 shows the results when using no denoising, DWT and Taut String.

When using Taut String denoising, the average of differences between the detected copy number values and the benchmark copy number values are $\% 25$ and $\% 36$ of the benchmark copy number values across all amplified and deleted genes, respectively. These averages when using no denoising and using DWT are shown in Table 5. Taut String outperforms DWT in providing more accurate copy number values as shown in Table 4 and Table 5. As can be seen in these tables, using denoising is beneficial in terms of improving accuracy of copy number values as well.

\section{Runtime comparison}

CBS segmentation is the most commonly used and effective segmentation methods; however, it is slow when readcount data are very noisy. This is because it uses an iterative algorithm based on the variance of the data. Denoising methods that smooths readcount data can help to speed up the segmentation by CBS. As we show in Fig. 1, Taut String generates smoother data with more clear edges (low fluctuations at breakpoints), which results in faster segmentation by CBS. In this section, we calculated the overall runtime of denoising and segmentation algorithm together using real and simulated data sets on a 64-bit Windows 10 Operating System, with intel core i7-7500 U 2.7 GHz CPU and 16 GB DDR4 memory. The time complexity of Taut String and DWT are $O(n)$ and $O(n \log n)$ respectively [70]. From the runtime perspective, applying Taut String and CBS algorithms subsequently surpasses applying DWT and CBS. Using the real data sets, on average, DWT and CBS combination took 76.35 min while Taut String and CBS combination took only $21.23 \mathrm{~min}$. We observed similar behavior using simulated data. On average, DWT and CBS took 30.67 s while Taut String and CBS took only $10.45 \mathrm{~s}$.

\section{Discussion}

Readcount data's noise and biases distort the association between copy numbers and read coverages. These biases and noise need to be removed from noisy readcount data in order to have more accurate $\mathrm{CNV}$ identification. In this study, we proposed to use a signal processing approach based on total variations and Taut String to reduce readcount noise. In general, denoising improves sensitivity and FDR of CNV detection. However, edge protecting denoising approaches (e.g. Taut String and DWT) significantly outperform regular denoising methods (e.g. MA). In fact, a denoising method that can generate less fluctuations and sharper edges at breakpoints, such as Taut String, leads to detecting more accurate CNVs compared to other methods (e.g. DWT and MA). Using simulated and real data, we showed that Taut String outperforms DWT and MA approaches in terms of sensitivity, FDR, and specificity in CNV detection. The major advantage of Taut String is its ability in preserving CNV segments' breakpoints, resulting in increasing the detection accuracy of a CNV segmentation method, especially in detecting narrow CNVs. Due to Taut String power in

Table 3 Overall Performance of The Denoising Methods Using the Real WES Data

\begin{tabular}{|c|c|c|c|c|c|c|}
\hline \multirow[t]{2}{*}{ Denoising Methods } & \multicolumn{3}{|c|}{ Amplified CNVs } & \multicolumn{3}{|c|}{ Deleted CNVs } \\
\hline & Sensitivity & FDR & Specificity & Sensitivity & FDR & Specificity \\
\hline Before applying denoising method & $50.99 \%$ & $42.06 \%$ & $80.45 \%$ & $60.37 \%$ & $64.32 \%$ & $56.71 \%$ \\
\hline After applying DWT & $68.81 \%$ & $41.65 \%$ & $79.92 \%$ & $77.65 \%$ & $54.32 \%$ & $72.23 \%$ \\
\hline After applying Taut String & $69.52 \%$ & $40.21 \%$ & $84.51 \%$ & $79.93 \%$ & $50.72 \%$ & $77.25 \%$ \\
\hline
\end{tabular}


Table 4 Percentage of CNV genes that the difference between their copy number values from their benchmark values are less than $20 \%$ of the benchmark copy number values

\begin{tabular}{lll}
\hline Denoising methods & Amplified genes & Deleted genes \\
\hline no denoising & $44.48 \%$ & $42.77 \%$ \\
DWT & $73.36 \%$ & $58.35 \%$ \\
Taut String & $76.87 \%$ & $70.26 \%$ \\
\hline
\end{tabular}

estimating piecewise constant signals, the identified CNV segments have more accurate breakpoints and copy number values. In addition, the Taut String method is very efficient. The complexity of its algorithm is linear in time. NGS data are big and using an efficient and fast CNV detection method is essential. The proposed method is an effective and practical approach to improve CNV identification due to its high efficiency and its power to detect true CNVs. However, adjusting the optimization parameter for Taut String, which indicates the upper bound and lower bound of error is challenging. In order to have a high accurate denoising approach, selecting an appropriate error bound is critical. Having a global error bond reduces the effectiveness of Taut String. Local squeezing that reduces the error bond locally by a constant factor improves the performance of Taut String. When this constant factor is small, the algorithm takes less time and many extreme values will be generated. In contrast, when this constant factor is close to one, we can have more accurate $\mathrm{CNV}$ detection while the computational time increases.

\section{Conclusions}

Denoising readcount data for $\mathrm{CNV}$ detection methods that are based on depth of coverage, can remarkably improve the accuracy of $\mathrm{CNV}$ detection. However, most of the current $\mathrm{CNV}$ detection tools do not employ denoising techniques, which results in low sensitivity and high false positive rates. Also, noise cancellation algorithms need to be very efficient in order to not increase the overall complexity of CNV identification. In this work, we developed an efficient and effective denoising method based on signal processing approaches. The proposed method uses the total variation approach for cancelling noise and employs the non-iterative, in-place Taut String algorithm to obtain the optimal approximation of denoised data. Signal processing

Table 5 Average differences between detected copy number values and benchmark copy number values respect to the benchmark copy number values

\begin{tabular}{lll}
\hline Denoising methods & Amplified genes & Deleted genes \\
\hline no denoising & $50 \%$ & $53 \%$ \\
DWT & $38 \%$ & $36 \%$ \\
Taut String & $25 \%$ & $36 \%$ \\
\hline
\end{tabular}

approaches have a long history in noise cancellation and can be extremely valuable for improving the accuracy of $\mathrm{CNV}$ detection. Selecting an appropriate denoising approach depends on the characteristics of the noise and signal. From a signal processing point of view, readcount data are sparse, discrete, and piecewise constant. Noise cancelation algorithms for these types of signals usually are Fourier based denoising methods and Kernel estimators. These techniques cannot separate spectra correctly. As a result, they can reduce noise, but are not able to preserve edges. By contrast, total variation denoising methods can simultaneously preserve edges, remove noise, and generate piecewise constant signals, even at high levels of noise. We used Taut String for efficient implementation of total variation denoising. To investigate the performance of the Taut String denoising approach, we compared the accuracy of detecting CNV segments when using Taut String denoising with those of when using DWT and MA denoising methods. DWT, which is commonly used in bioinformatics, is also an efficient and nonlinear smoothing method. However, this study showed that Taut String outperform DWT in both efficiency and accuracy.

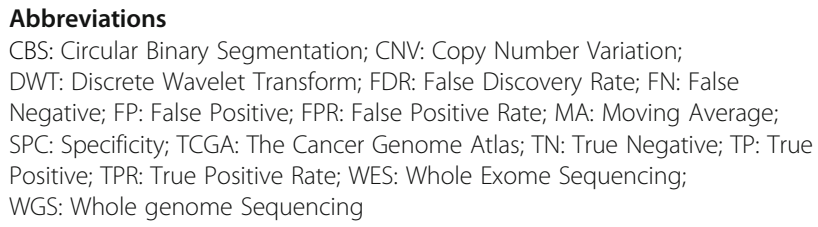

Acknowledgments

Not applicable.

Funding

Publication of this manuscript was sponsored by a grant from the National Institutes of Health (NIH, ROOLM011595, Pl: Nabavi).

\section{Availability of data and materials}

The breast cancer CNV data that are used as benchmark are available at TCGA's data portal (https://portal.gdc.cancer.gov/projects/TCGA-BRCA) The BAM files of the breast cancer data are protected data and are available at The Cancer Genomics Hub (CGHub), https://cghub.ucsc.edu/index.html. The simulator for generating simulated WES data is available at https:// github.com/NabaviLab/CNV-Sim.

\section{About this supplement}

This article has been published as part of BMC Bioinformatics Volume 19 Supplement 11, 2018: Proceedings from the 6th Workshop on Computational Advances in Molecular Epidemiology (CAME 2017). The full contents of the supplement are available online at https://bmcbioinformatics.biomedcentral. com/articles/supplements/volume-19-supplement-11.

\section{Authors' contributions}

SN, and FZ designed the study. FZ implemented the analysis; SN, FZ interpreted the results. SN, FZ and $\mathrm{AH}$ wrote the manuscript. All authors read and approved the final version of the manuscript.

Ethics approval and consent to participate

No ethics approval was required for the study. Input data are publicly available through The Cancer Genome Atlas (TCGA) and are simulated data.

Consent for publication

Not applicable. 


\section{Competing interests}

The authors declare that they have no competing interests.

\section{Publisher's Note}

Springer Nature remains neutral with regard to jurisdictional claims in published maps and institutional affiliations.

\section{Author details}

${ }^{1}$ Computer Science and Engineering Department, University of Connecticut, Storrs, CT, USA. ${ }^{2}$ Computer Science and Engineering Department and Institute for Systems Genomics, University of Connecticut, Storrs, CT, USA.

\section{Published: 22 October 2018}

\section{References}

1. lafrate AJ, Feuk L, Rivera MN, Listewnik ML, Donahoe PK, Qi Y, et al. Detection of large-scale variation in the human genome. Nat Genet. 2004; 36:949-51.

2. Sebat J, Lakshmi B, Troge J, Alexander J, Young J, Lundin P, et al. Largescale copy number polymorphism in the human genome. Science. 2004; 305:525-8

3. Zarrei M, MacDonald JR, Merico D, Scherer SW. A copy number variation map of the human genome. Nat Rev Genet. 2015;16:172-83.

4. Freeman $\mathrm{J}$, Perry GH, Feuk L, Redon R, McCarroll SA, Altshuler DM, et al. Copy number variation: new insights in genome diversity. Genome Res. 2006;16:949-61.

5. Feuk L, Carson AR, Scherer SW. Structural variation in the human genome. Nat Rev Genet. 2006;7:85-97.

6. Stranger BE, Forrest MS, Dunning M, Ingle CE, Beazley $C$, Thorne $N$, et al. Relative impact of nucleotide and copy number variation on gene expression phenotypes. Science. 2007:315:848-53.

7. Zhang F, Gu W, Hurles ME, Lupski JR. Copy number variation in human health, disease, and evolution. Annu Rev Genomics Hum Genet. 2009:10: 451-81.

8. Stankiewicz P, Lupski JR. Structural variation in the human genome and its role in disease. Annu Rev Med. 2010;61:437-55.

9. Shlien A, Malkin D. Copy number variations and cancer. Genome Med. 2009; $1: 62$.

10. Rashid NU, Giresi PG, Ibrahim JG, Sun W, Lieb JD. ZINBA integrates local covariates with DNA-seq data to identify broad and narrow regions of enrichment, even within amplified genomic regions. Genome Biol. 2011;12: R67.

11. Laird PW. Principles and challenges of genome-wide DNA methylation analysis. Nat Rev Genet. 2010;11:191.

12. Carter NP. Methods and strategies for analyzing copy number variation using DNA microarrays. Nat Genet. 2007:39:S16-21.

13. Metzker ML. Sequencing technologies - the next generation. Nat Rev Genet. 2010;11:31-46.

14. Ku CS, Loy EY, Salim A, Pawitan Y, Chia KS. The discovery of human genetic variations and their use as disease markers: past, present and future. J Hum Genet. 2010;55:403-15.

15. Teo SM, Pawitan Y, Ku CS, Chia KS, Salim A. Statistical challenges associated with detecting copy number variations with next-generation sequencing. Bioinformatics. 2012;28:2711-8

16. Duan J, Zhang J-G, Deng H-W, Wang Y-P. Comparative studies of copy number variation detection methods for next-generation sequencing technologies. PLoS One. 2013;8:e59128.

17. Tan R, Wang Y, Kleinstein SE, Liu Y, Zhu X, Guo H, et al. An evaluation of copy number variation detection tools from whole-exome sequencing data. Hum Mutat. 2014;35:899-907.

18. Zare F, Dow M, Monteleone N, Hosny A, Nabavi S. An evaluation of copy number variation detection tools for cancer using whole exome sequencing data. BMC Bioinformatics. 2017;18:286.

19. Zhao M, Wang Q, Wang Q, Jia P, Zhao Z. Computational tools for copy number variation (CNV) detection using next-generation sequencing data: features and perspectives. BMC bioinformatics. 2013;14(11):S1.

20. Pirooznia M, Goes FS, Zandi PP. Whole-genome CNV analysis: advances in computational approaches. Front Genet. 2015;6 https://doi.org/10.3389/ fgene.2015.00138
21. do Nascimento F, Guimaraes KS. Copy Number Variations Detection: Unravelling the problem in tangible aspects. IEEE/ACM transactions on computational biology and bioinformatics. 2017;14(6):1237-50.

22. Yoon S, Xuan Z, Makarov V, Ye K, Sebat J. Sensitive and accurate detection of copy number variants using read depth of coverage. Genome Res. 2009; 19:1586-92.

23. Xi R, Lee S, Park PJ. A survey of copy-number variation detection tools based on high-throughput sequencing data. Curr Protoc Hum Genet Editor Board Jonathan Haines Al 2012; Chapter 7:Unit7.19.

24. Abyzov A, Urban AE, Snyder M, Gerstein M. CNVnator: an approach to discover, genotype, and characterize typical and atypical CNVs from family and population genome sequencing. Genome Res. 2011;21:974-84.

25. Boeva V, Zinovyev A, Bleakley K, Vert J-P, Janoueix-Lerosey I, Delattre O, et al. Control-free calling of copy number alterations in deep-sequencing data using GC-content normalization. Bioinformatics. 2011;27:268-9.

26. Chiang DY, Getz G, Jaffe DB, O'Kelly MJT, Zhao X, Carter SL, et al. Highresolution mapping of copy-number alterations with massively parallel sequencing. Nat Methods. 2009;6:99-103.

27. Ivakhno S, Royce T, Cox AJ, Evers DJ, Cheetham RK, Tavare S. CNAseg--a novel framework for identification of copy number changes in cancer from second-generation sequencing data. Bioinformatics. 2010;26:3051-8.

28. Klambauer G, Schwarzbauer K, Mayr A, Clevert D-A, Mitterecker A, Bodenhofer U, et al. Cn.MOPS: mixture of Poissons for discovering copy number variations in next-generation sequencing data with a low false discovery rate. Nucleic Acids Res. 2012;40:e69.

29. Krumm N, Sudmant PH, Ko A, O'Roak BJ, Malig M, Coe BP, et al. Copy number variation detection and genotyping from exome sequence data. Genome Res. 2012;22:1525-32.

30. Magi A, Benelli M, Yoon S, Roviello F, Torricelli F. Detecting common copy number variants in high-throughput sequencing data by using JointSLM algorithm. Nucleic Acids Res. 2011;39:e65.

31. Miller CA, Hampton O, Coarfa C, Milosavljevic A. ReadDepth: a parallel R package for detecting copy number alterations from short sequencing reads. PLoS One. 2011;6:e16327.

32. Sathirapongsasuti JF, Lee H, Horst BAJ, Brunner G, Cochran AJ, Binder S, et al. Exome sequencing-based copy-number variation and loss of heterozygosity detection: ExomeCNV. Bioinformatics. 2011;27:2648-54.

33. Xi R, Hadjipanayis AG, Luquette LJ, Kim T-M, Lee E, Zhang J, et al. Copy number variation detection in whole-genome sequencing data using the Bayesian information criterion. Proc Natl Acad Sci U S A. 2011;108:E1128-36.

34. Xie C, Tammi MT. CNV-seq, a new method to detect copy number variation using high-throughput sequencing. BMC Bioinformatics. 2009;10:80.

35. Minoche AE, Dohm JC, Himmelbauer H. Evaluation of genomic highthroughput sequencing data generated on Illumina HiSeq and genome analyzer systems. Genome Biol. 2011;12:R112.

36. Kozarewa I, Ning Z, Quail MA, Sanders MJ, Berriman M, Turner DJ. Amplification-free Illumina sequencing-library preparation facilitates improved mapping and assembly of $(\mathrm{G}+\mathrm{C})$-biased genomes. Nat Methods. 2009:6:291-5.

37. Dohm JC, Lottaz C, Borodina T, Himmelbauer H. Substantial biases in ultrashort read data sets from high-throughput DNA sequencing. Nucleic Acids Res. 2008;36:e105

38. Rieber N, Zapatka M, Lasitschka B, Jones D, Northcott P, Hutter B, et al. Coverage Bias and sensitivity of variant calling for four whole-genome sequencing technologies. PLoS One. 2013;8:e66621.

39. lakovishina D, Janoueix-Lerosey I, Barillot E, Regnier M, Boeva V. SV-bay: structural variant detection in cancer genomes using a Bayesian approach with correction for GC-content and read mappability. Bioinformatics. 2016 32:984-92.

40. Boeva V, Popova T, Bleakley K, Chiche P, Cappo J, Schleiermacher G, et al. Control-FREEC: a tool for assessing copy number and allelic content using next-generation sequencing data. Bioinformatics. 2011;28:423-5.

41. Benjamini Y, Speed TP. Summarizing and correcting the GC content bias in high-throughput sequencing. Nucleic Acids Res. 2012; https://doi.org/10. 1093/nar/gks001.

42. Benjamin DJ, Cesarini D, van der Loos MJ, Dawes CT, Koellinger PD, Magnusson PK, et al. The genetic architecture of economic and political preferences. Proc Natl Acad Sci. 2012;109:8026-31.

43. Liao C, Yin A, Peng C, Fu F, Yang J, Li R, et al. Noninvasive prenatal diagnosis of common aneuploidies by semiconductor sequencing. Proc Natl Acad Sci. 2014;111:7415-20. 
44. Gusnanto A, Wood HM, Pawitan Y, Rabbitts P, Berri S. Correcting for cancer genome size and tumour cell content enables better estimation of copy number alterations from next-generation sequence data. Bioinformatics. 2012:28:40-7.

45. Koboldt DC, Zhang Q, Larson DE, Shen D, McLellan MD, Lin L, et al. VarScan 2: somatic mutation and copy number alteration discovery in cancer by exome sequencing. Genome Res. 2012;22:568-76.

46. Lai D, Ha G, Shah S, Lai MD, biocViews Sequencing P, CopyNumberVariation M. Package "HMMcopy." 2011.

47. Amarasinghe KC, Li J, Hunter SM, Ryland GL, Cowin PA, Campbell IG, et al. Inferring copy number and genotype in tumour exome data. BMC Genomics. 2014;15:732.

48. Stamoulis C, Betensky RA. A novel signal processing approach for the detection of copy number variations in the human genome. Bioinformatics. 2011:27:2338-45.

49. Ben-Yaacov E, Eldar YC. A fast and flexible method for the segmentation of aCGH data. Bioinformatics. 2008;24:1139-45.

50. Hsu L, Self SG, Grove D, Randolph T, Wang K, Delrow JJ, et al. Denoising array-based comparative genomic hybridization data using wavelets. Biostatistics. 2005;6:211-26.

51. Condat L. A direct algorithm for 1-D Total variation Denoising. IEEE Signal Process Lett. 2013;20:1054-7.

52. Belle A, Ansari S, Spadafore M, Convertino VA, Ward KR, Derksen H, et al. A signal processing approach for detection of hemodynamic instability before decompensation. PLoS One. 2016;11:e0148544.

53. Dümbgen L, Kovac A. Others. Extensions of smoothing via taut strings. Electron J Stat. 2009;3:41-75.

54. Davies PL, Kovac A. Local extremes, runs, strings and multiresolution. Ann Stat. 2001;1:1-48.

55. Kovac A. Smooth functions and local extreme values. Comput Stat Data Anal. 2007:51:5155-71.

56. Zare F, Ansari S, Najarian K, Nabavi S. Noise cancellation for robust copy number variation detection using next generation sequencing data. IEEE. 2017:230-6. https://doi.org/10.1109/BIBM.2017.8217654.

57. Olshen $A B$, Venkatraman $E$, Lucito R, Wigler M. Circular binary segmentation for the analysis of array-based DNA copy number data. Biostatistics. 2004;5: 557-72.

58. Quinlan AR, Hall IM. BEDTools: a flexible suite of utilities for comparing genomic features. Bioinformatics. 2010;26:841-2.

59. Aird D, Ross MG, Chen W-S, Danielsson M, Fennell T, Russ C, et al. Analyzing and minimizing PCR amplification bias in lllumina sequencing libraries. Genome Biol. 2011;12:R18.

60. Gao F, Zhang C-T. GC-profile: a web-based tool for visualizing and analyzing the variation of GC content in genomic sequences. Nucleic Acids Res. 2006; 34(suppl 2):W686-91.

61. Donoho DL. De-noising by soft-thresholding. IEEE Trans Inf Theory. 1995;41: 613-27.

62. Schall O, Belyaev A, Seidel H-P. Robust filtering of noisy scattered point data. IEEE. 2005:71-144. https://doi.org/10.1109/PBG.2005.194067.

63. Donoho DL. Nonlinear wavelet methods for recovery of signals, densities, and spectra from indirect and noisy data. In: In proceedings of Symposia in applied mathematics: American Mathematical Society; 1993. p. 173-205.

64. Cho H, Fryzlewicz P. Multiscale interpretation of taut string estimation and its connection to unbalanced Haar wavelets. Stat Comput. 2011;21:671-81.

65. Duan J, Zhang J-G, Lefante J, Deng H-W, Wang Y-P. Detection of copy number variation from next generation sequencing data with total variation penalized least square optimization. IEEE. 2011:3-12. https://doi.org/10.1109/ BIBMW.2011.6112348.

66. Barbero A, Sra S. Modular proximal optimization for multidimensional totalvariation regularization. 2014. arXiv preprint arXiv:1411.0589.

67. Li H, Durbin R. Fast and accurate short read alignment with burrowswheeler transform. Bioinformatics. 2009;25:1754-60.

68. GenomicRanges. Bioconductor. http://bioconductor.org/packages/ GenomicRanges/. Accessed 9 Oct 2017.

69. Lawrence M, Huber W, Pages H, Aboyoun P, Carlson M, Gentleman R, et al. Software for computing and annotating genomic ranges. PLoS Comput Biol. 2013;9:e1003118.

70. Pique-Regi R, Ortega A, Tewfik A, Asgharzadeh S. Detecting changes in DNA copy number: reviewing signal processing techniques. IEEE Signal Process Mag. 2012;29:98-107.

Ready to submit your research? Choose BMC and benefit from:

- fast, convenient online submission

- thorough peer review by experienced researchers in your field

- rapid publication on acceptance

- support for research data, including large and complex data types

- gold Open Access which fosters wider collaboration and increased citations

- maximum visibility for your research: over $100 \mathrm{M}$ website views per year

At BMC, research is always in progress.

Learn more biomedcentral.com/submissions 